\title{
Is rosuvastatin better than atorvastatin and simvastatin in the prevention of in-stent restenosis and atherosclerosis progression in patients after superficial femoral artery stenting due to chronic lower limb ischaemia? The preliminary case-control study
}

\author{
Jacek Budzynski ${ }^{1,2}$, Joanna Kubiak², Grzegorz Pulkowski², Karol Suppan², Marcin Wasielewski², \\ Joanna Wisniewska², Radoslaw Wieczor ${ }^{2}$ \\ 'Department of Vascular and Internal Diseases, Faculty of Health Sciences, Ludwik Rydygier Collegium Medicum in Bydgoszcz, \\ Nicolaus Copernicus University in Torun, Poland \\ ${ }^{2}$ Clinic of Vascular and Internal Diseases, Jan Biziel University Hospital No. 2 in Bydgoszcz, Poland \\ This paper was presented during $6^{\text {th }}$ International Scientific-Educational Conference of the Polish Angiological Society, Warsaw, 23-24 October 2015
}

Abstract

Introduction. Statins effect on the outcome of endovascular intervention due to chronic lower limb ischaemia (CLLI) is still uncertain. The aim of this study was to determine the effect of statin type on the late outcome of superficial femoral artery (SFA) stenting in patients with CLLI.

Material and methods. Retrospective analysis of the medical documentation of 275 consecutive patients treated with SFA stenting due to CLLI, including I 25 (45\%) patients with critical limb ischaemia (CLI). Measured outcomes were: target lesion revascularization (TLR), target extremity revascularization (TER), and target limb amputation (TLA).

Results. Statins were used by 267 (97\%) of the patients, respectively: atorvastatin ( $n=191,70 \%)$, simvastatin $(n=31,11 \%)$ and rosuvastatin $(n=45,16 \%)$. During the $675.0 \pm 569.7$ days of follow-up, TLR was required by 79 (29\%) patients, TER by 109 (39\%), and TLA by 27 (10\%). Patients treated with rosuvastatin in comparison with those treated with atorvastatin, in spite of greater initial LDL and triglyceride levels, required $\operatorname{TER}(p=0.01)$ and $\operatorname{TLR}(p=0.03)$ less frequently. The risk of TER in patients treated with rosuvastatin was significantly $(p=0.016)$ lower than in individuals treated with atorvastatin and simvastatin, as shown in the Kaplan-Meier analysis. Cox's proportional hazards regression showed that therapy with rosuvastatin was the strongest factor (HR $0.40 \pm 95 \%$ Cl; 0.2-0.8I) decreasing the likelihood of TER.

Conclusions. Rosuvastatin after SFA stenting seems to have the strongest effect on reduction in reintervention risk but without influence on limb salvage.

Key words: chronic lower limb ischaemia, statins, endovascular therapy, superficial femoral artery stenting, reintervention risk, amputation risk

Acta Angiol 2016; 22, I: 5-11

Address for correspondence: Jacek Budzynski, Department of Vascular and Internal Diseases, Faculty of Health Sciences, Ludwik Rydygier Collegium Medicum in Bydgoszcz, Nicolaus Copernicus University in Torun, Poland, e-mail: budz@cps.pl 


\section{Introduction}

Chronic lower limb ischaemia (LLI) is a chronic disorder, which progresses in about $20-30 \%$ of cases, leading to the shortening of claudication distance to a level that limits everyday activity and/or to symptoms of critical limb ischaemia (CLI) with rest pain and sleep disturbance, necrosis and the need for amputation [I]. These symptoms mainly decrease quality of life. However, they are also related to increased mortality due to cardiovascular comorbidities in the other vascular beds. These facts determine the aims of the treatment of patients with LLI. These aims are mainly a reduction in all-cause and cardiovascular mortality, limb salvage and improvement in symptoms severity and quality of life through the lengthening of claudication distance, which allows for increased daily activity and improved function. These aims can be achieved by lifestyle modification and control of the atherosclerosis risk factors (smoking cessation, blood pressure control), pharmacotherapy, walking training, and revascularization (endovascular, surgical or hybrid) [I-3]. The main problem with endovascular treatment is the recurrence of symptoms due to in-stent restenosis (ISR) and/or atherosclerosis progression. A number of procedural methods have been proposed to decrease the risk of ISR or to treat it when occurs. They are as follows: a) the use of a drug-eluting stent (DES) which releases anti-proliferative drugs, statins, cytokine and growth factor inhibitors, as well as the use of covered stents; or b) avoidance of stent implantation by the use of a drug-eluting balloon (DEB), a cutting balloon or debulking techniques (for various types of atherectomy) [2-8]. However, the role of pharmacotherapy for this purpose is not well known [3]. In the context of the effect of statins on decreasing ISR incidence in patients after coronary artery stenting $[9,10]$ and their diverse hypolipidemic and pleiotropic effects [1 I, I2], we tried to assess their efficacy in patients after superficial femoral artery (SFA) stenting.

\section{Material and methods}

We conducted a retrospective analysis of the medical documentation (obtained during hospitalization and visits in our out-patients clinic) of 275 all, consecutive patients treated in our clinic due to chronic LLI with SFA stenting as a first revascularization approach between I January 20 II and 3 I December 20 I4. After percutaneous revascularization all patients were followed-up in our out-patients clinic having performed duplex scan of treated limb. The inclusion criteria were as follows: SFA stenting, lack of previous vascular interventions in stented extremity, and presence on at least one visit in our out-patient clinic in 2015. The following data were analysed: demographic (e.g. age and gender), clinical (e.g. symptom severity), biochemical (e.g. complete blood count, creatinine, total cholesterol, low-density lipoprotein (LDL) and high-density lipoprotein (HDL) cholesterol, triglycerides and blood glucose) and angiographic (TransAtlantic Inter-Society Consensus [TASC] classification of femoropopliteal lesions) [I, 2].

\section{Measured outcomes}

During the period between the performance of the percutaneous procedure and 3 I August 20 I5, the following efficacy outcomes of the analysis were taken: target lesion revascularization (TLR), target extremity revascularization (TER), and target limb amputation (TLA) [13]. TLR was defined as repeat percutaneous (endovascular) revascularization for a lesion anywhere within a stent or the $5 \mathrm{~mm}$ border proximal or distal to the stent due to symptom recurrence and target lesion occlusion or significant narrowing (mainly due to ISR). TER was defined using a wider set of criteria than usual [13], as the revascularization of a previously stented lower limb due to the target lesion or a new lesion at least $10 \mathrm{~mm}$ outside the proximal or distal border of the stent due to symptom recurrence (claudication impeding the normal daily functioning of the patient or critical limb ischaemia) and lesion diameter stenosis $\geq 50 \%$ (an effect of atherosclerosis progression). TLA was defined as a minor or major amputation of a previously stented limb during the follow-up period.

The following safety outcomes were also analysed: rhabdomyolysis, acute liver injury, and other adverse reactions to statin treatment.

\section{Bioethics}

The study protocol was approved by the local Bioethics Committee (agreement number KBI39/20I4). All procedures were conducted in compliance with the Declaration of Helsinki.

\section{Statistical analysis}

A licensed version of STATISTICA (a data analysis software system), version 10 (StatSoft, Inc., 20I I) was used. The normal distribution of the study variables was checked using the Shapiro-Wilk test. The results were mainly presented as the mean \pm the standard deviation (SD) or $\mathrm{n}, \%$. The statistical significance of the differences between the patient groups was verified in relation to the type of variable distribution using parametric tests (Student's t-test, $\mathrm{Chi}^{2}$ ) if the distribution of the variables was normal, and non-parametric tests (Mann-Whitney $U, \mathrm{Chi}^{2}$ ) if not. The association between the type of statin used and end-point occurrence 
Table I. Demographic and clinical characteristics of patients studied in relation to the statin taken $(n=267,97 \%)$

\begin{tabular}{|c|c|c|c|}
\hline Feature & $\begin{array}{l}\text { Atorvastatin } \\
(n=191)\end{array}$ & $\begin{array}{l}\text { Simvastatin } \\
(n=3 I)\end{array}$ & $\begin{array}{l}\text { Rosuvastatin } \\
(n=45)\end{array}$ \\
\hline Age (years) & $65.4 \pm 10.2$ & $65.4 \pm 8.6$ & $64.3 \pm 9.5$ \\
\hline Male gender (n, \%) & $123(64 \%)$ & $23(74 \%)$ & $31(69 \%)$ \\
\hline Smoking habit (n, \%) & $99(52 \%)$ & $14(45 \%)$ & $21(47 \%)$ \\
\hline Hypertension (n, \%) & $136(7 \mid \%)$ & $22(7 \mid \%)$ & $39(87 \%)$ \\
\hline Diabetes mellitus (n, \%) & $71(37 \%)$ & $14(45 \%)$ & $13(28 \%)$ \\
\hline Dyslipidaemia (n, \%) & I5I (79\%) & $21(68 \%)$ & $4 \mid(9 \mid \%)^{\#}$ \\
\hline $\mathrm{BMI}\left[\mathrm{kg} / \mathrm{m}^{2}\right]$ & $27.2 \pm 5.1$ & $31.4 \pm 3.1$ & $24.2 \pm 2.1$ \\
\hline CAD (n, \%) & 77 (40\%) & $19(61 \%)^{*}$ & $23(51 \%)$ \\
\hline Stroke (n, \%) & $16(8 \%)$ & $7(23 \%)^{*}$ & I (2\%) \\
\hline $\mathrm{CHF}(\mathrm{n}, \%)$ & $49(26 \%)$ & $10(32 \%)$ & $8(18 \%)$ \\
\hline Creatinine $>2 \mathrm{mg} / \mathrm{dL}(\mathrm{n}, \%)$ & $6(3 \%)$ & I (3\%) & $0(0 \%)$ \\
\hline Total cholesterol [mg/dL] & $183.8 \pm 46.0$ & $166.0 \pm 28.8$ & $243.8 \pm 55.5^{\#}$ \\
\hline LDL cholesterol [mg/dL] & $122.6 \pm 39.1$ & $112.9 \pm 36.0$ & $138.1 \pm 48.9^{\#}$ \\
\hline HDL cholesterol [mg/dL] & $48.4 \pm 9.4$ & $49.3 \pm 8.5$ & $61.0 \pm 18.4$ \\
\hline Triglycerides [mg/dL] & $144.3 \pm 75.9$ & $138.5 \pm 84.0$ & $181.9 \pm 85.3^{\#}$ \\
\hline Fibrate in combination therapy $(\mathrm{n}, \%)$ & $8(4 \%)$ & I (3\%) & $3(7 \%)$ \\
\hline Rutherford classification & $4.0 \pm 1.2$ & $3.8 \pm 1.1$ & $3.7 \pm 1.2$ \\
\hline$A B I$ of the treated leg & $0.53 \pm 0.18$ & $0.58 \pm 0.16$ & $0.54 \pm 0.14$ \\
\hline $\begin{array}{l}\text { Classification of the treated lesion according to } \\
\text { TASC-II (B/C/D; n, \%) }\end{array}$ & $\begin{array}{c}24(13 \%) / \\
2(59 \%) / \\
55(29 \%)\end{array}$ & $\begin{array}{c}8(26 \%) / \\
14(45 \%) / \\
9(29 \%)\end{array}$ & $\begin{array}{c}8(18 \%) / \\
29(64 \%) / \\
8(18 \%)\end{array}$ \\
\hline Number of implanted stents (n) & $\mathrm{I} .5 \pm 0.8$ & $1.3 \pm 0.9$ & $1.6 \pm 0.75$ \\
\hline Sum of stent lengths [mm] & $95.2 \pm 62.1$ & $89.5 \pm 72.4$ & $100.6 \pm 66.1$ \\
\hline $\operatorname{TLR}(\mathrm{n}, \%)$ & $58(30 \%)$ & II (35\%) & $9(20 \%)^{\#}$ \\
\hline TER (n, \%) & $82(43 \%)$ & $14(45 \%)$ & $10(22 \%)^{\#}$ \\
\hline TLA (n, \%) & $18(9 \%)$ & $5(16 \%)$ & $2(4 \%)$ \\
\hline Number of days between intervention and TLR & $358.6 \pm 400.9$ & $239.8 \pm 240.6$ & $407.8 \pm 313.6$ \\
\hline Number of days between intervention and TER & $262.9 \pm 343 \pm 340.8$ & $340.8 \pm 210.5$ & $389.0 \pm 271.4$ \\
\hline Number of days between intervention and TLA & $657.0 \pm 585.4$ & $711.2 \pm 503.1$ & $541.6 \pm 518.4$ \\
\hline
\end{tabular}

BMI — body mass index; CAD — coronary artery disease; CHF — chronic heart failure; LDL — low-density lipoprotein; HDL — high-density lipoprotein; ABI — ankle-brachial index; TASC — TransAtlantic Inter-Society Consensus; TLR — target lesion revascularization; TER — target extremity revascularization; TLA — target limb amputation. Statistical significance of difference $(\mathrm{p}<0.05)$ : *between atorvastatin and simvastatin groups; * between atorvastatin and rosuvastatin groups

was verified using survival analysis, the Cox's F test, the log-rank test in the Kaplan-Meier method for two groups (rosuvastatin vs. atorvastatin vs. simvastatin), as well as using Cox's proportional hazard regression analysis. A p-value $<0.05$ was considered statistically significant.

\section{Results}

In the analysed group, 125 (45\%) patients underwent SFA stenting due to CLI and I50 (55\%) due to intermittent claudication limiting usual daily physical activity. In whole group the severity and prevalence of respective atherosclerosis risk factors during hospitalization linked with endovascular procedure were: age (65 \pm 9.8 years), male gender $(\mathrm{n}=181,66 \%)$, dyslipidaemia ( $n=218,79 \%)$, hypertension $(n=202,73 \%)$, smoking habit $(n=135,49 \%)$, and diabetes mellitus $(n=101$, $37 \%)$. Statins were recommended in discharge cards in 267 (97\%) patients: atorvastatin in 191 (69\%), which included I $5 \mathrm{I}(55 \%)$ patients with a recommended dose of $40 \mathrm{mg}$; simvastatin $40 \mathrm{mg}$ for 31 (1 $1 \%$ ) patients, and rosuvastatin $20 \mathrm{mg}$ for 45 patients (16\%). All the patients for whom statins were recommended declared adherence to the treatment during control visits in out-patients clinic. A comparison of demographic and clinical data for the groups of patients using the respective types of statins is presented in Table I. 
Table 2. Cox's proportional hazard regression for the risk of target extremity revascularization $\left(\right.$ Chi $\left.^{2}=58.48 ; p=0.00002\right)$

\begin{tabular}{|c|c|c|c|c|c|}
\hline Feature & $\beta$ & $\begin{array}{l}\text { Standard } \\
\text { error of } \beta\end{array}$ & $\begin{array}{c}\text { Wald } \\
\text { statistics }\end{array}$ & $\mathbf{p}$ & $\begin{array}{c}\text { Hazard ratio } \\
\pm 95 \% \mathrm{Cl}\end{array}$ \\
\hline Age (years) & -0.04 & 0.01 & 10.23 & 0.001 & $0.96 \pm 0.94-0.99$ \\
\hline Male gender $(n, \%)$ & 0.21 & 0.23 & 0.85 & 0.36 & $1.23 \pm 0.79-1.94$ \\
\hline Smoking habit (n, \%) & -0.06 & 0.22 & 0.08 & 0.78 & $0.94 \pm 0.62-1.44$ \\
\hline Hypertension (n, \%) & -0.30 & 0.25 & 1.42 & 0.23 & $0.74 \pm 0.45-1.22$ \\
\hline Diabetes mellitus (n, \%) & 0.48 & 0.24 & 4.07 & 0.04 & $1.62 \pm 1.01-2.58$ \\
\hline Dyslipidaemia (n, \%) & -0.10 & 0.29 & 0.12 & 0.73 & $0.90 \pm 0.51-1.6$ \\
\hline BMI $\left[\mathrm{kg} / \mathrm{m}^{2}\right]$ & -0.28 & 0.09 & 9.74 & 0.002 & $0.76 \pm 0.63-0.90$ \\
\hline CAD (n, \%) & 0.31 & 0.26 & 1.42 & 0.23 & $1.35 \pm 0.6-1.52$ \\
\hline Stroke (n, \%) & -0.48 & 0.42 & 1.29 & 0.26 & $0.62 \pm 0.27-1.42$ \\
\hline $\mathrm{CHF}(\mathrm{n}, \%)$ & 0.44 & 0.28 & 2.52 & 0.11 & $1.56 \pm 0.90-2.7$ \\
\hline Creatinine $>2$ mg/dL (n, \%) & -1.73 & 1.05 & 2.72 & 0.1 & $0.18 \pm 0.02-1.38$ \\
\hline Total cholesterol [mg/dL] & -0.002 & 0.005 & 0.10 & 0.75 & $0.99 \pm 0.98-1.01$ \\
\hline LDL cholesterol [mg/dL] & 0.006 & 0.003 & 2.86 & 0.09 & $1.01 \pm 0.99-1.01$ \\
\hline HDL cholesterol [mg/dL] & 0.01 & 0.027 & 0.24 & 0.62 & $1.01 \pm 0.96-1.07$ \\
\hline Triglycerides [mg/dL] & -0.002 & 0.003 & 1.14 & 0.29 & $0.99 \pm 0.99-1.0$ \\
\hline Rutherford classification & 0.38 & 0.09 & 18.44 & 0.0001 & $1.46 \pm 1.23-1.74$ \\
\hline $\mathrm{ABI}$ of treated leg & -1.39 & 1.23 & 1.28 & 0.26 & $0.25 \pm 0.02-2.78$ \\
\hline $\begin{array}{l}\text { Classification of the treated lesion } \\
\text { according to TASC-II (B/C/D; n, \%) }\end{array}$ & -0.21 & 0.17 & 1.48 & 0.22 & $0.81 \pm 0.58-1.14$ \\
\hline Number of implanted stents (n) & 0.25 & 0.19 & 1.81 & 0.18 & $1.28 \pm 0.89-1.84$ \\
\hline Sum of stent lengths [mm] & 0.002 & 0.002 & 1.24 & 0.27 & $1.00 \pm 0.99-1.01$ \\
\hline Rosuvastatin vs. other statins & -0.91 & 0.36 & 6.43 & 0.01 & $0.40 \pm 0.2-0.81$ \\
\hline
\end{tabular}

$\mathrm{Cl}$ — confidence interval; BMI — body mass index; CAD — coronary artery disease; CHF — chronic heart failure; LDL — low-density lipoprotein; HDL — high-density lipoprotein; ABI — ankle-brachial index; TASC — TransAtlantic Inter-Society Consensus

During on average $675.0 \pm 569.7$ days of follow-up (median \pm low-upper quartile: $630 \pm 168-1036$ days), TLR was required by 79 (29\%), TER by $109(40 \%)$, and TLA by 27 (10\%) patients. Patients treated with rosuvastatin, in spite of higher levels of LDL cholesterol and triglycerides at the day of first endovascular procedure compared with individuals treated with atorvastatin, less frequently required TER $(p=0.0 \mathrm{I})$ and had fewer reinterventions considered as TER ( $22 \%$ vs. $43 \%$; $p=0.03$ ) (Tab. I). The prevalence of TLR and TLA in the rosuvastatin group of patients was non-significantly lower than in patients treated with other statins (the combined group of atorvastatin and simvastatin) (Tab. I). In the survival analysis $\left(\mathrm{Chi}^{2}=8.2 ; \mathrm{p}=0.016\right)$, the risk of TER in patients treated with rosuvastatin was significantly lower than in patients with other hypolipidaemic therapy (Fig. I). Cox's proportional hazard regression showed that therapy with rosuvastatin was one of the few independent significant variables and the strongest factor $(0.40 \pm 0.2-0.8 \mathrm{I})$ decreasing the risk of TER in the two-year follow-up (Tab. 2).

In the medical documentation available for the patients studied, we did not find any information showing the occurrence of any safety outcomes that might be related to therapy with statins.

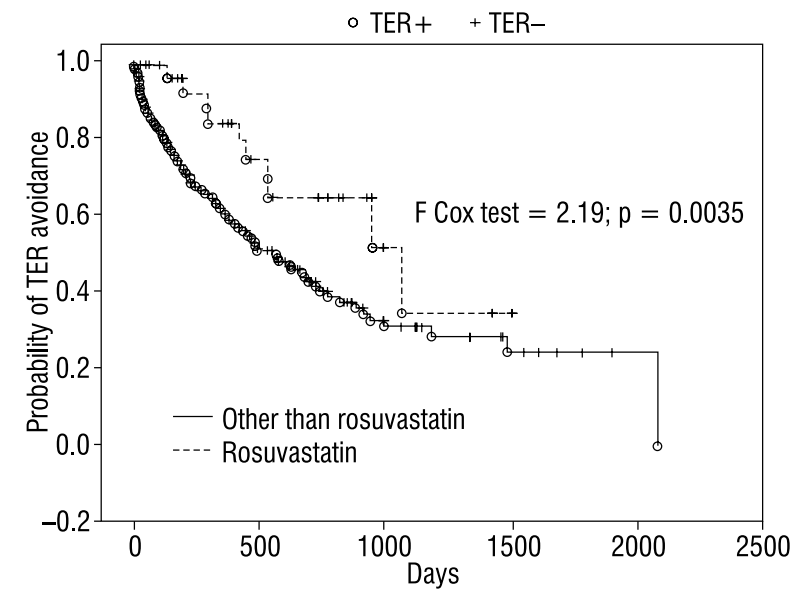

Figure I. The effect of rosuvastatin therapy compared with other statins on the risk of target extremity revascularization (TER) following endovascular intervention according to Kaplan-Meier analysis

\section{Discussion}

In our case-control retrospective analysis of medical documentation of patients treated with SFA stenting 
due to various severity of chronic LLI, we estimated the effect of the type of statin used on primary stent patency and the need for revascularization. In other words, we checked the influence of statins on ISR and atherosclerosis progression in patients after SFA stenting. In the analysed period, $29 \%$ of the patients needed TLR due to loss of primary stent patency in the course of ISR, and $39 \%$ required the revascularization of a previously stented extremity (TER), which demonstrated that in $10 \%$ of the individuals during the two-year observation the clinically important progression of atherosclerosis occurred in the extra-stent part of the vascular bed. Our results concerning TLR prevalence are similar to other reports, which showed that clinically important ISR occurred in as many as I8-40\% of patients treated during the first year after stent implantation $[14,15]$.

In our study, in spite of differences in the number of subjects taking the various types of statins, we found that patients from the rosuvastatin group had a lower risk of reintervention (TLR and TER), which, in relation to TER, was confirmed by survival analysis (Fig. I, Tab. 2). Such observations suggest an advantage of this statin over the other hypolipidaemic drugs in the prevention of ISR (TLR) and atherosclerosis progression (TER). As patients treated with rosuvastatin had the highest LDL blood concentration at the time of the first endovascular procedure (Tab. I), the results obtained may be explained by the stronger pleiotropic effect of this statin, which, among other things, depends on an improvement in endothelial function, endothelial regeneration, increased nitric oxide synthesis (vasodilatation promotion) and angiogenic effect, reduced oxidant stress, inflammation suppression, as well as atherosclerosis plaque stabilization [3, 12]. Rosuvastatin has also revealed an anti-inflammatory and anti-thrombotic effect [16], which is highly important in patients with LLI, the majority of whom have increased levels of the inflammatory biomarkers linked to ISR prevalence [17].

In some previous investigations, the association between statin use and the patency of arteries in the lower extremities at various vascular levels after endovascular intervention were examined [3, 18-25], but we did not find any data concerning the advantage of using rosuvastatin in the prevention of standard end-point occurrence. Some of these works showed that in patients with intermittent claudication and CLI treated endovascularly statin use was mainly linked with a decrease in all-cause and cardiovascular mortality, as well as cardiovascular event (acute coronary syndrome, stroke or death) $[3,19,20]$, and a smaller number of patients showed a lower risk of undesirable end-points related to the treated extremity, such as symptom severity, the need for reintervention, and amputation (limb events, limb morbidity) [3, 2I-24]. For example, Dosluoglu et al. [25] showed that of $717 \mathrm{pa}-$ tients following endovascular treatment due to LLI, statin users $(55.4 \%)$ had better survival in comparison to their counterparts, but without significant differences in stent patency. A review of earlier publications concerning the effect of statins both on general cardiovascular end-points and on the effectiveness of the endovascular treatment of LLI was presented by Erez and Leitersdorf [18] and Bonaca and Creager [3].

In our patients with chronic LLI, statin use (97\%) was more prevalent than in other studies (18.7\% [3], $67 \%$ [20] and 55.4\% [25], respectively). On the other hand, our study showed that, in spite of the widely known and above-mentioned favourable effects of statins, the level of LDL cholesterol recommended for patients with very high cardiovascular risk was not achieved in the majority of our patients with LLI. In our study, the mean LDL blood concentration amounted to $124 \mathrm{mg} / \mathrm{dL}$, although a study by Westin et al. [22] showed that patients with LDL level $>130 \mathrm{mg} / \mathrm{dL}$ had increased risk of a combined cardiovascular event and mortality compared with patients with lower levels of LDL. The European Society for Cardiology recommends lowering LDL cholesterol to $<2.5 \mathrm{mmol} / \mathrm{L}(100 \mathrm{mg} / \mathrm{dL})$ for patients with LLI, and optimally to $<1.8 \mathrm{mmol} / \mathrm{L}$ $(70 \mathrm{mg} / \mathrm{dL})$ or $\leq 50 \%$ of the initial LDL cholesterol level when reaching a target therapeutic LDL blood concentration is impossible [26]. Adherence to these therapeutic aims is better in patients with coronary artery disease (CAD) than in subjects with LLI [3]. In a study by Pereg et al. [27], an LDL level $<100 \mathrm{mg} / \mathrm{dL}$ was achieved in $65 \%$ vs. $47 \%(p<0.0001)$ of CAD and LLI patients respectively, and LDL level $<70 \mathrm{mg} / \mathrm{dL}$ was achieved in $23 \%$ vs. $13 \%$ ( $p<0.0001$ ) of CAD and LLI patients, respectively. On the other hand, in Heart Protection Study the effect of simvastatin was independent of initial LDL cholesterol blood concentration and other atherosclerosis risk factors [3].

Unfortunately, as most authors, we could not avoid some methodological shortcomings that could have influenced the strength of the deductions based on our results. Firstly, our study was performed according to a retrospective medical documentation analysis, and without randomization to the respective statin treatment, on a relatively small patient group, and with the lack of a control group (only $3 \%$ of the subjects did not take any statins). However, it would not have been ethical to perform any investigation on patients with cardiovascular disease who are not treated with statins. Secondly, we did not know the hypolipidaemic effect of the recommended statins in patients in whom a renewed endovascular procedure was not necessary. We based our results only on the information provided by patients during monitoring visits, but a recent publication has shown that only $54.3 \%$ of patients 
adhered to their prescription for anti-platelet therapy after percutaneous coronary interventions, in spite of their declared adherence to the recommended therapy [28]. Thirdly, in our group of patients the potential confounding factors were not balanced (Tab. I). On the one hand, patients treated with rosuvastatin had significantly greater prevalence of dyslipidaemia, as well as had higher total, LDL cholesterol, and triglycerides blood concentrations, what might act to the disadvantage of this statin. On the other hand, they had slightly but not significantly lower prevalence of diabetes, past stroke, congestive heart failure and renal insufficiency, as well as lover BMI value, which could negatively affect the outcomes of atorvastatin compared with rosuvastatin. Nevertheless, we think that our observation of rosuvastatin having the most beneficial effect in the prevention of ISR and atherosclerosis progression in patients with LLI after endovascular intervention is worthy of further evaluation. The importance of such investigations increases in the context of the favourable effect of the use of biodegradable stents eluting rosuvastatin in animal models [29].

\section{Conclusions}

I. Rosuvastatin seems to have the most favourable clinical effect on the risk of reintervention after SFA stenting, but without influence on limb salvage. However, this positive outcome needs to be confirmed due to the small size of the group investigated and imbalance of the potential confounding factors.

2. No satisfactory control of lipid-related atherosclerosis risk factors was found in our study in patients with chronic LLI.

\section{Conflict of interest}

None declared.

\section{References}

I. Norgren L, Hiatt WR, Dormandy JA et al (2007) Inter-Society Consensus for the Management of Peripheral Arterial Disease (TASC II). Eur J Vasc Endovasc Surg; 33 (Suppl. I): SI-75.

2. TASC Steering Committee, Jaff MR, White CJ et al (2015) An update on methods for revascularization and expansion of the TASC lesion classification to include below-the-knee arteries: A supplement to the Inter-Society Consensus for the Management of Peripheral Arterial Disease (TASC II). J Endovasc Ther; 22: 663-677. doi: 10.1177//5266028/5592206.

3. Bonaca MP, Creager MA (2015) Pharmacological treatment and current management of peripheral artery disease. Circ Res; 116 : 1579-1598. doi: 10.1 161/CIRCRESAHA.I14.303505.

4. Tosaka A, Soga Y, Lida $O$ et al (2012) Classification and clinical impact of restenosis after femoropopliteal stenting. J Am Coll Cardiol; 59: 16-23.
5. Razzouk L, Aggarwal S, Gorgani F et al (2013) In-stent restenosis in the superficial femoral artery. Ann Vasc Surg; 27: 510-524.

6. Liistro F, Grotti S, Porto I et al (2013) Drug-eluting balloon in peripheral intervention for the superficial femoral artery: the DEBATE-SFA randomized trial (drug eluting balloon in peripheral intervention for the superficial femoral artery). JACC Cardiovasc Interv; 6: I295-1302.

7. Swanson N, Hogrefe K, Javed Q et al (2003) Vascular endothelial growth factor (VEGF)-eluting stents: in vivo effects on thrombosis, endothelialization and intimal hyperplasia. J Invasive Cardiol; 15: 688-692.

8. Gouëffic Y, Kaladji A, Guyomarch B et al (2014) Bare metal stent versus paclitaxel eluting stent for intermediate length femoropopliteal arterial lesions (BATTLE trial): study protocol for a randomized controlled trial. Trials; 15: 423.

9. Johnson C, Waters DD, DeMicco DA et al (2008) Comparison of effectiveness of atorvastatin $10 \mathrm{mg}$ versus $80 \mathrm{mg}$ in reducing major cardiovascular events and repeat revascularization in patients with previous percutaneous coronary intervention (post hoc analysis of the Treating to New Targets [TNT] Study). Am J Cardiol; 102: |3|2-13|7.

10. Prasad K (2013) Do statins have a role in reduction/prevention of post-PCl restenosis? Cardiovasc Ther; 31: 12-26. doi: 10.1 III/j. 1755-5922.2011.00302.x.

II. Hatzitolios Al, Athyros VG, Karagiannis A et al. (2009) Implementation of strategy for management for overt dyslipidemia: The IMPROVE-dyslipidemia study. Int J Cardiol; 134: 322-329.

12. Vaughan CJ, Murphy MB, Buckley BM (1996) Statins do more than just lower cholesterol. Lancet; 348: 1079-1082.

13. Diehm N, Baumgartner I, Jaff $M$ et al (2007) A call for uniform reporting standards in studies assessing endovascular treatment for chronic ischaemia of lower limb arteries. Eur Heart j; 28: 798-805.

14. Schillinger M, Sabeti S, Loewe C et al (2006) Balloon angioplasty versus implantation of nitinol stents in the superficial femoral artery. N Engl J Med; 354: 1879-1888.

15. Laird JR, Katzen BT, Scheinert D et al (2010) Nitinol stent implantation versus balloon angioplasty for lesions in the superficial femoral artery and proximal popliteal artery: twelve-month results from the RESILIENT randomized trial. Circ Cardiovasc Interv; 3: 267-276.

16. Ridker PM, Danielson E, Fonseca FA et al (2008) Rosuvastatin to prevent vascular events in men and women with elevated c-reactive protein. N Engl J Med; 359: 2195-2207.

17. McDermott MM, Lloyd-Jones DM (2009) The role of biomarkers and genetics in peripheral arterial disease. J Am Coll Cardiol; 54: 1228-1237.

18. Erez G, Leitersdorf E (2007) The rationale for using HMG-CoA reductase inhibitors ('statins') in peripheral arterial disease. Eur J Vasc Endovasc Surg; 33: 192-201.

19. Suckow BD, Kraiss LW, Schanzer A et al (2015) Statin therapy after infrainguinal bypass surgery for critical limb ischemia is associated with improved 5-year survival. J Vasc Surg; 61: I26-133. doi: 10.1016/j.jvs.2014.05.093.

20. Armstrong EJ, Chen DC, Westin GG et al (2014) Adherence to guideline-recommended therapy is associated with decreased major adverse cardiovascular events and major adverse limb events among patients with peripheral arterial disease. J Am Heart Assoc; 3: e000697. doi: 10.1161/JAHA. I 13.000697. 
21. Aiello FA, Khan AA, Meltzer AJ et al (2012) Statin therapy is associated with superior clinical outcomes after endovascular treatment of critical limb ischemia. J Vasc Surg; 55: 37I-379.

22. Westin GG, Armstrong EJ, Bang $\mathrm{H}$ et al (2014) Association between statin medications and mortality, major adverse cardiovascular event, and amputation-free survival in patients with critical limb ischemia. J Am Coll Cardiol; 63: 682-690.

23. Kumbhani DJ, Steg PG, Cannon CP et al (2014) Statin therapy and long-term adverse limb outcomes in patients with peripheral artery disease: insights from the REACH registry. Eur Heart J; 35: 2864-2872.

24. Tomoi $Y$, Soga $Y$, lida $O$ et al (2013) Efficacy of statin treatment after endovascular therapy for isolated below-the-knee disease in patients with critical limb ischemia. Cardiovasc Interv Ther; 28: 374-382. doi: 10.1007/s 12928-013-0188-6.

25. Dosluoglu HH, Davari-Farid S, Pourafkari $L$ et al (2014) Statin use is associated with improved overall survival without affecting patency and limb salvage rates following open or endovascular revascularization. Vasc Med; 19: 86-93.
26. Hirsch AT, Haskal ZJ, Hertzer NR et al (2006) ACC/AHA guidelines for the management of patients with peripheral arterial disease (lower extremity, renal, mesenteric, and abdominal aortic): a collaborative report from the American Association for Vascular Surgery/Society for Vascular Surgery, Society for Cardiovascular Angiography and Interventions, Society for Vascular Medicine and Biology, Society of Interventional Radiology, and the ACC/AHA Task Force on Practice Guidelines (Writing Committee to Develop Guidelines for the Management of $\mathrm{Pa}$ tients With Peripheral Arterial Disease). JACC; 47: 1239-1312.

27. Pereg D, Neuman Y, Elis A (20I2) Comparison of lipid control in patients with coronary versus peripheral artery disease following the first vascular intervention. Am J Cardiol; I I0: 1266-1269.

28. Kubica A, Kasprzak M, Obońska K et al (2015) Discrepancies in assessment of adherence to antiplatelet treatment after myocardial infarction. Pharmacology; 95: 50-58.

29. Lee CH, Chang SH, Lin YH (2014) Acceleration of re-endothelialization and inhibition of neointimal formation using hybrid biodegradable nanofibrous rosuvastatin-loaded stents. Biomaterials; 35: 44I7-4427. doi: 10.1016/j.biomaterials.2014.02.017. 